\title{
Genetics Corner: Townes-Brocks Syndrome in an Infant with Familial Imperforate Anus
}

Robin Clark, MD, Subhadra Ramanathan, MS.

\section{Case summary:}

A genetic consultation was requested for a term male infant with a preauricular tag and an imperforate anus. He was born at 38 weeks six days gestation to a 22-year old G2P1 mother by repeat C-section at a local community hospital. The pregnancy was planned, and routine prenatal care began at nine weeks gestation. The mother denied all teratogenic exposures, including alcohol, tobacco, and drug use. The birth weight was 3739 grams, appropriate for gestational age. The baby was transferred to our tertiary care NICU on the day of birth for imperforate anus. Minimal meconium was noted on the perineum from an apparent fistula. An echocardiogram revealed a patent foramen ovale with a small shunt and bidirectional flow across the atrial septum. The Head ultrasound exam showed mild asymmetry of the lateral ventricles, left larger than right without sonographic evidence of intracranial hemorrhage. Spine ultrasound and radiographs were normal. A renal ultrasound exam showed minimal right renal pelvocaliectasis.

The physical exam showed an alert, active infant with mild hypertelorism. The left ear had an overfolded helix superiorly and a bifurcated ear tag on the tragus. The extremities, specifically the thumbs, were normal. There was a small tuft of hair at the base of the sacrum. The phallus, testes, and scrotum were normal. The anus was imperforate.

The baby underwent a posterior sagittal anorectoplasty without complications. He passed the newborn hearing screen and was discharged. When he was seen in a follow-up visit at two months of age, he was feeding and gaining weight well and stooling normally. His parents were using a Hegar dilator. His development was age-appropriate.

\section{Family history:}

The family history was significant for imperforate anus in the patient's 23-year old father, who was otherwise well. A 3-year old sister was healthy without ear or anal anomalies. There was no other significant family history of birth defects, developmental delay, intellectual disability, early infant deaths, or multiple miscarriages. Parents reported Hispanic (father) and Hispanic and Caucasian (mother) ancestry. They denied consanguinity.

\section{Clinical assessment:}

Because of the family history of anal atresia in the father and the presence of minor anomalies involving the ear and other organs in this infant, the genetics consultant proposed Townes-Brocks syndrome as the likely diagnosis and suggested SALL1 gene testing with reflex testing for a clinical exome sequencing test if the SALL1 study were normal.

\section{Laboratory studies:}

Chromosome microarray was normal: $\operatorname{arr}(1-22) \times 2,(X Y) \times 1$.

Gene analysis for SALL1 included gene sequencing and deletion/duplication analysis. Results showed a heterozygous likely pathogenic variant in SALL1: c.3160C>T (p.Arg1054*). This variant causes a premature translational stop signal and a truncated protein product signified by the asterisk in the protein (p.) variant nomenclature.

\section{Genetic counseling:}

Townes-Brocks syndrome (TBS, OMIM 107480) is an autosomal dominant, multiple congenital anomaly syndrome that includes imperforate anus. It is characterized by the triad of the imperforate anus (84\%), dysplastic ears $(87 \%)$, and thumb malformations $(89 \%)$. Hearing impairment, both sensorineural and conductive, can be seen in about $65 \%$ of affected individuals. Renal impairment, including end-stage renal disease, may occur with or without structural abnormalities in about $40 \%$. Rare findings include eye anomalies (coloboma, Duane anomaly). Intellectual disability is not common and is seen in only $10 \%$ of affected individuals (Kohlhase et al., 2016).

The clinical features of TBS are variable, even among affected individuals from the same family. This infant has no thumb or other major anomalies, and the father had only imperforate anus without ear anomalies or other signs of TBS. This illustrates that there can be overlap between the milder expression of syndromic disorders and the nonsyndromic forms of congenital malformations

Penetrance and expression are terms used to describe the variable effects of single-gene disorders. Penetrance describes the proportion of individuals in a population who carry a specific gene variant and express any degree of the disease-related phenotype. Penetrance is "complete" when all individuals with a pathogenic variant in the responsible gene have physical features of that disorder; such is the case with achondroplasia. Penetrance is "reduced" or "incomplete" when some individuals with the genetic variant do not have any expected physical signs. Reduced penetrance occurs in familial cancer syndromes caused by variants in BRCA1 or BRCA2. Nonpenetrance is complete when all individuals with the gene variant have a normal phenotype. Nonpenetrance could describe unaffected heterozygous carriers of a gene variant that, when homozygous, causes an autosomal recessive disorder.

Although penetrance of the TBS phenotype has been reported to be complete in individuals with heterozygous SALL1 variants, there is clinical variability among affected individuals with TBS, which clinical geneticists describe as variable "expression." TBS features can vary widely, encompassing what might look like isolated and apparently nonsyndromic ear anomalies or imperforate anus. With this in mind, it was surprising to find that this particular SALL1 variant had been previously reported, in a homozygous state, to cause an autosomal recessive disorder quite unlike TBS and with nonpenetrance in the apparently unaffected heterozygous relatives. Two affected siblings who were homozygous for this same SALL1 variant had multiple congenital anomalies and intellectual disabilities (Vodopiutz et al., 2013). This family's affected children's phenotype included tetralogy of Fallot, polycystic hypoplastic kidneys with prenatal onset of chronic renal failure, limb and ear malformations, and sensorineural deafness corpus callosum hypoplasia, cortical blindness, and lack of psychomotor

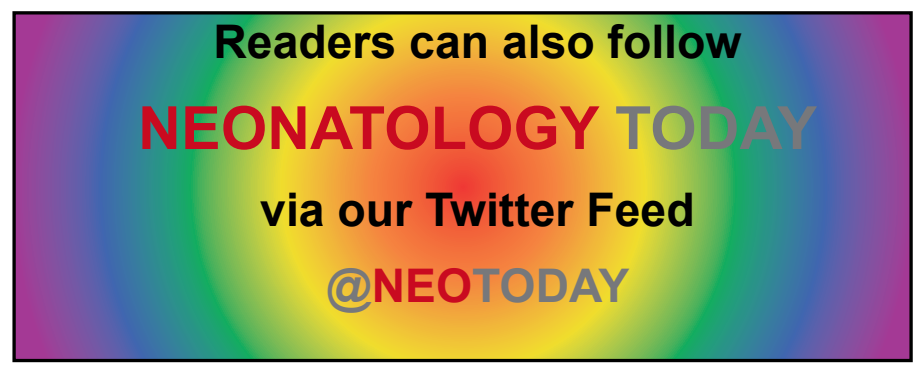




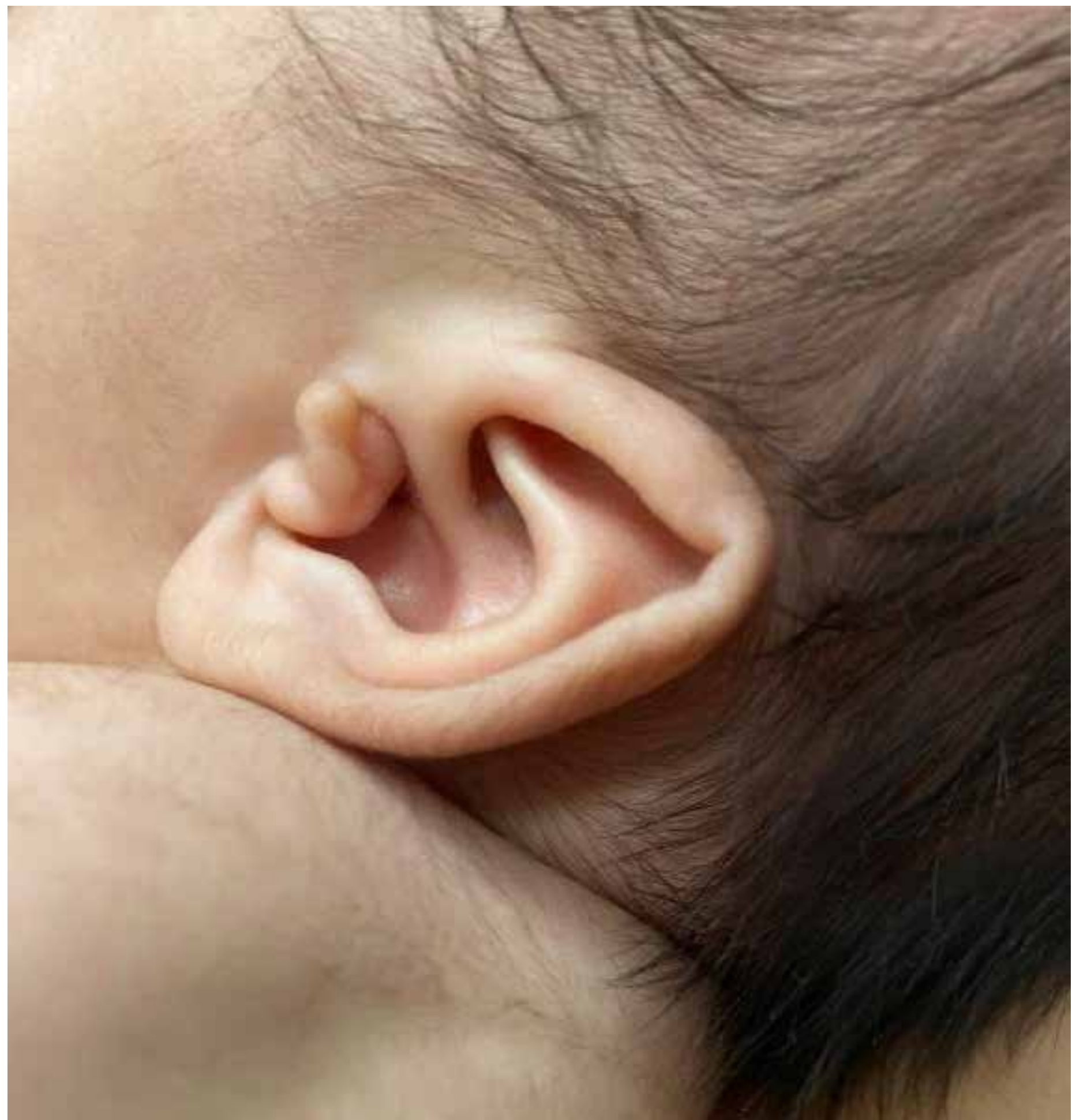

Graphic 1: This infant with Townes-Brocks syndrome has minor anomalies in the left ear's helical folding that also has a bifurcated tag on the tragus.

The only worldwide monthly publication
exclusively serving Pediatric and Adult
Cardiologists that focus on Congenital/
$\begin{aligned} & \text { Structural Heart Disease (CHD), and } \\ & \text { CARDIOLOGY } \\ & \text { TODAY }\end{aligned}$


development. Importantly, none of the twelve heterozygous carriers from this family had features of TBS. This report suggests that this variant results in a weaker and less pathogenic "hypomorphic" allele that causes disease in the homozygous state and that it may be nonpenetrant in heterozygous "carriers." However, the same variant has also been reported in a heterozygous proband and a heterozygous parent with clinical features that suggested TBS (ClinVar Variation ID: 219151).

It is possible that a hypomorphic allele, such as this SALL1 variant, could result in such a mild expression of TBS that it could be interpreted as a nonsyndromic imperforate anus, as seems to be the case in our patient's father and an attenuated TBS phenotype in this infant. We postulate that the patient inherited his SALL1 variant from his father, but this has not yet been confirmed. We recommended targeted testing for this SALL1 variant in the patient's father. If testing confirms that he is heterozygous (or mosaic) for this variant, the recurrence risk for imperforate anus in his future offspring could be as high as 50\%. Given the lack of penetrance of this gene variant in the family reported in 2013, we also recommended targeted variant testing in the patient's unaffected sister.

\section{Practical applications:}

1. Always take a careful family history as it may offer the key to the diagnosis

2. Take note of minor anomalies. Even those that are not functionally important may suggest a pattern of anomalies associated with a particular syndrome.

3. Understand that incomplete penetrance and variable expression can blur the line between a single gene syndromic disorder with a higher recurrence risk and a nonsyndromic, typically multifactorial congenital anomaly with lower recurrence risk.

4. Appreciate that some pathogenic variants may produce gene products with some residual function. Such "hypomorphic" alleles for syndromic disorders may contribute to the familial nature of some nonsyndromic congenital anomalies.

\section{Reference:}

1. Kohlhase J. Townes-Brocks Syndrome. 2007 Jan 24 [Updated 2016 Jan 14]. In: Adam MP, Ardinger HH, Pagon RA, et al., editors. GeneReviews ${ }^{\circledR}$ [Internet]. Seattle (WA): University of Washington, Seattle; 1993-2020. Available from: https://www.ncbi.nlm.nih.gov/books/NBK1445/

2. Vodopiutz J, Zoller H, Fenwick AL, et al. Homozygous SALL1 mutation causes a novel multiple congenital anomaly-mental retardation syndrome. J Pediatr. 2013 Mar;162(3):612-7. PMID: 23069192.

The authors have no relevant disclosures.

NT

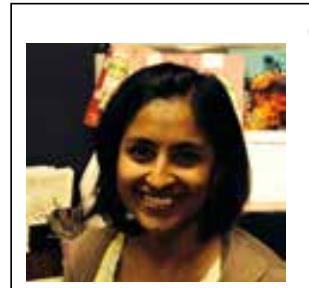

Corresponding Author

Subhadra (Subha) Ramanathan, M.Sc., M.S.

Licensed and Certified Genetic Counselor

Assistant Professor, Pediatrics

Loma Linda University Health

2195 Club Center Drive, Ste A

San Bernardino, CA 92408

SRamanathan@llu.edu

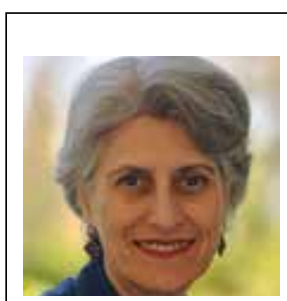

Robin Clark, MD

Professor, Pediatrics

Loma Linda University School of Medicine

Division of Genetics

Department of Pediatrics

Email rclark@llu.edu

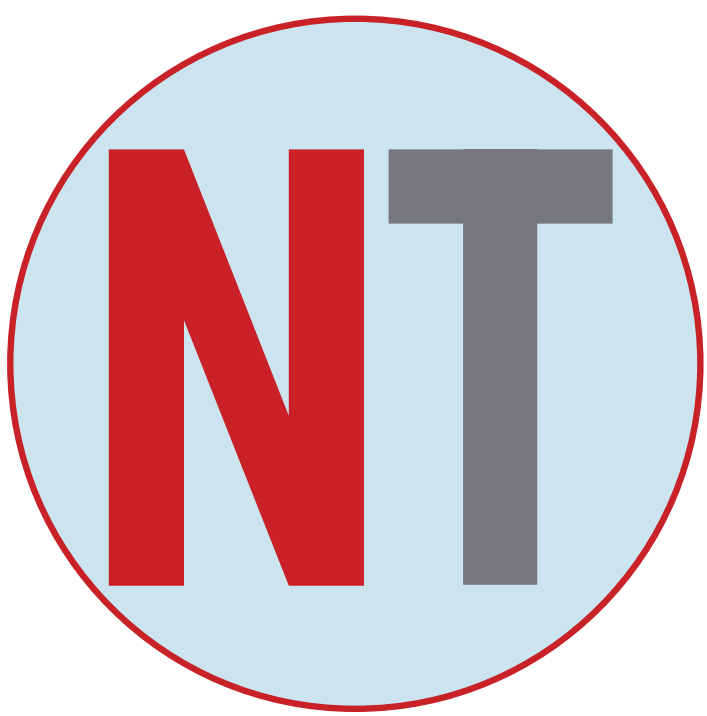

NEONATOLOGY TODAY is interested in publishing manuscripts from Neonatologists, Fellows, NNPs and those involved in caring for neonates on case studies, research results, hospital news, meeting announcements, and other pertinent topics.

Please submit your manuscript to: LomaLindaPublishingCompany@gmail.com 